\title{
Current practices in scientific poster preparation - survey results
}

Helena Jambor, Mildred Scheel Early Career Center, Medical Faculty, Technische Universität Dresden, Germany, helena.jambor@tu-dresden.de, ORCID: 0000-0003$3397-1842$

\begin{abstract}
Poster sessions allow conference participants to discuss recent research and are a first public discussion for many early career scientists. Preparing a poster that is both accessible and insightful to the audience is key. Yet often both poster presenter and audiences complain about overloaded and incomprehensible posters, despite many guides and how-to papers being available. Here, I probed the current practices of poster preparation and present the results of an online survey and in-person interviews with poster presenters. This revealed, that the on average scientists spend two days on the preparation of a poster and receive neither training nor feedback on this task. Early feedback, provided during the drafting stage of the poster preparation process, could safe many hours of work, free valuable time for research, and help making research reach a larger audience.
\end{abstract}

\section{Introduction}

Visualizations are central in scientific communication. At conferences, poster presentations are a key event, especially when participant numbers exceed the speaker slots. At large conferences thousands of posters may be presented: 2500 at the meeting of the American Society for Cell Biology, 3000 at the meeting of the American Society for Human Genetics, and 1500 at the TAG meeting, the Technology Association of Grantmakers. Poster sessions allow attendees to discuss data prior to publication and are considered essential training for early career scientists. For many $\mathrm{PhD}$ students, a poster session is their first experience in publicly presenting results to a wider audience.

The format of all scientific posters is similar, and includes a header with title and authors information, an overview of the rationale, methods, and results, and at times references, acknowledgements, and summaries. Text and visualizations such as schematics, images, charts, and diagrams are juxtaposed much more than in a traditional paper. Many universities provide poster templates with a pre-set layout. Over the years, numerous guides for poster preparation were published. Brown (1996, 1997) is a classic that is regularly cited and passed around. Boullata (2007), Gundogan (2016) and Jambor (2017) provide updates with an exemplar template (Boullata), details on text, font size, information flow and the importance of feedback (Gunogan) and tips for layout (Jambor). Erren and Bourne, 2007, expand from the 
display and also provide recommendations for the actual presentation and having an "elevator pitch" ready. A black humoured account is given by Thomas G. Wolcott and Jambor provided a "Bad Poster Bingo" game for your next conference.

Although poster sessions are widespread and provide a wonderful opportunity for indepth discussion, they are often subject of complaints and participation can be low. One reason is certainly that even today posters are often not self-explanatory. Instead, they still have too much text, cryptic abbreviations, inconsistent colours and a lack of visual order. To understand how poster legibility may be improved, I conducted a qualitative survey of how scientists design their posters. Many scientists require a considerable amount of time to prepare a poster, in part due to a lack of training in appropriate software and in visual design principles. In addition, most posters appear to be prepared without feedback from colleagues, which presents a missed opportunity for quick poster improvement.

\section{Method and Material}

Questions of the online-poll: Country of studies, Subject of studies, Previous training in poster making, Number of posters prepared, Time spend on poster preparation, Time spend on poster design, Number of people involved in poster preparation/their role, Poster designed from scratch/template.

Questions of the in-person interview: Gender [Male/female, categorical], Career stage [Predoc/Postdoc/PI, categorical], Country of studies, Subject of studies, Time for poster preparation $[<1 \mathrm{~h}, 1$ day, $>1$ day, categorical], Time spend on poster design [none, $<1 \mathrm{~h},>1 \mathrm{~h}$, categorical], Software used for poster preparation,

Number of people involved in poster preparation/their role, Poster designed from scratch/template, Previous training in poster making, Personal critique of poster (what went well, what was difficult/frustrating).

23 interviews were conducted at the "Complex Life of mRNA" conference held at the EMBL in Heidelberg in October 2016. Online poll was circulated via social media and by Email.

\section{Results}

\section{Online Survey}

To survey scientific poster design broadly, I performed an online poll among a diverse group of scientists working in the life sciences (summarized in Figure 1). This group included 90 scientists with undergraduate training from 26 countries. Half of the group studied biology/related subjects, the other half included physicist, engineers, chemists and computer scientists. Around 20\% had received some training in statistical data representation, yet only 6 of the 90 respondents stated that they had been trained in the graphical aspects of number representation and/or design principles for information design. 
During their academic careers, respondents had already prepared a median of 8 posters. They estimated that each poster took two to three working days in preparation time, with about half this time, one to one-and-a-half days, exclusively devoted to design aspects. For poster preparation, most of which were prepared without an existing template, $51 \%$ of the online polled participants used vector-based graphic software (Adobe Illustrator, CorelDraw, Inkjet) and 31\% used PowerPoint. The majority of those polled had either received no feedback or only limited feedback from just one co-author. (Figure 1)

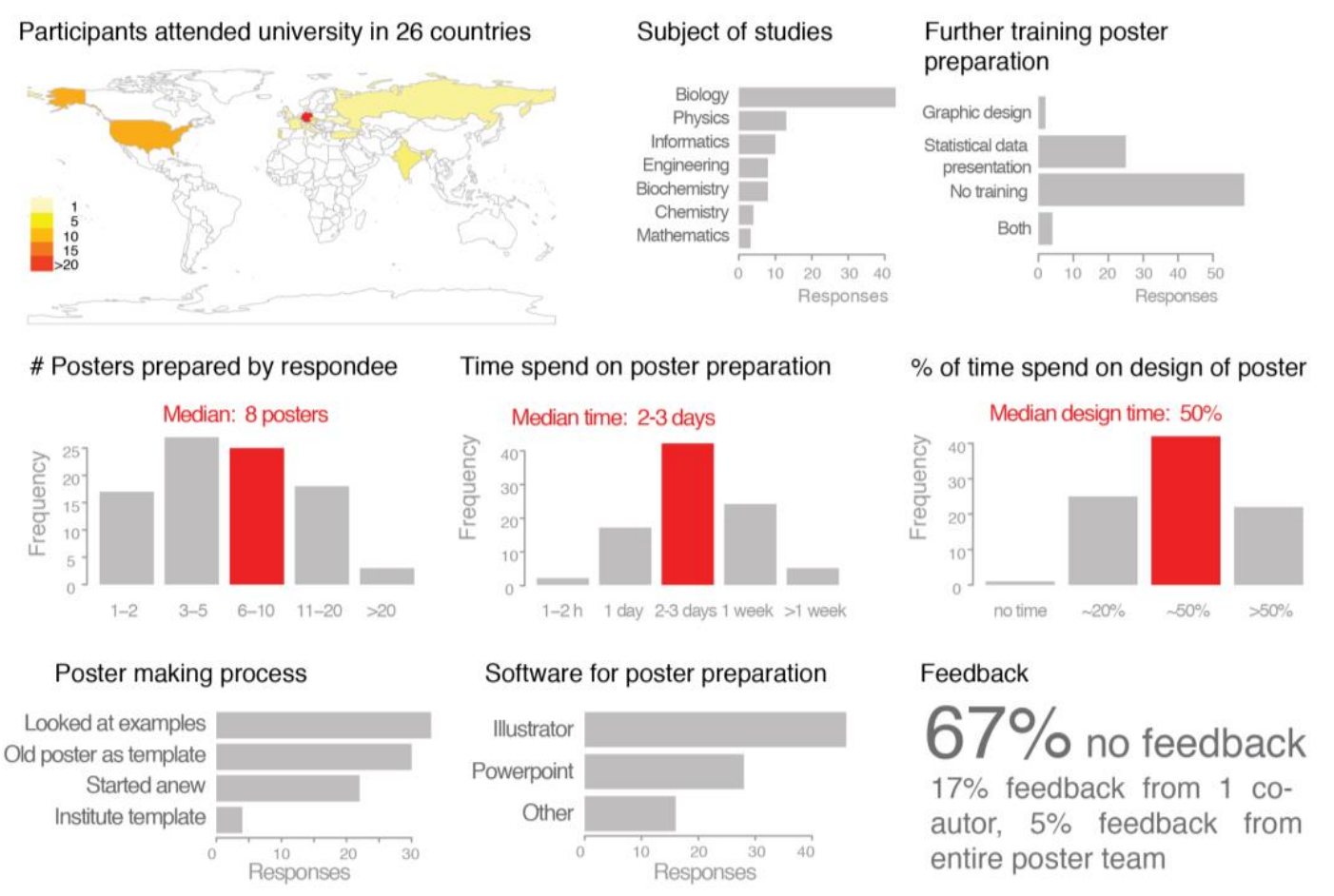

Figure 1 Online survey data

\section{Interviews}

To get more insight into the process of poster presentation, I also interviewed 23 scientists in person during the poster session of a scientific conference in Heidelberg. The 23 scientists were randomly sampled (whoever happen to be at their poster during the poster session) and covered almost $10 \%$ of the posters presented at the meeting. The group included roughly equal numbers of male and female scientists. The majority of respondents, $21 / 23$, had studied biology or a closely related field and they had received undergraduate training in fifteen different countries. Only one person had received training in graphical representation of statistical data. Not one respondent was trained in graphic design principles or poster preparation during either undergraduate or graduate education (Figure 2).

The career stages were diverse with two principal investigators, thirteen postdocs, and ten $\mathrm{PhD}$ students. On average the interviewees had prepared around eight posters in their scientific life, but many had prepared substantially more, up to 35 posters. The 
median time for preparing the poster was one to two days, with half of the time spend on poster design aspects including layout, colours, and text arrangement/fonts etc. (Figure 2).

When discussing the process of poster presentation, it emerged that most interviewees $(17 / 23)$ had not started their poster from scratch, but instead recycled an older poster of theirs or a colleague. In most cases this template was a PowerPoint file, which was also the software most used for poster preparation for 13 participants, while 10 used a vector-graphic software (Figure 2).

Some interviewees, especially those who had prepared posters with PowerPoint, expressed frustration that images resolution suffered after scaling the slide to poster size. Correspondingly, several participants stated that they lacked the time to learn using a vector-based software and had not been given a chance for a training in appropriate software. One person stated that the poster process took almost a week because they used the poster preparation as a chance to learn Illustrator.

Similar to the online responses, the interviewees had usually not received feedback on their poster preparation from either co-workers or their principal investigator.
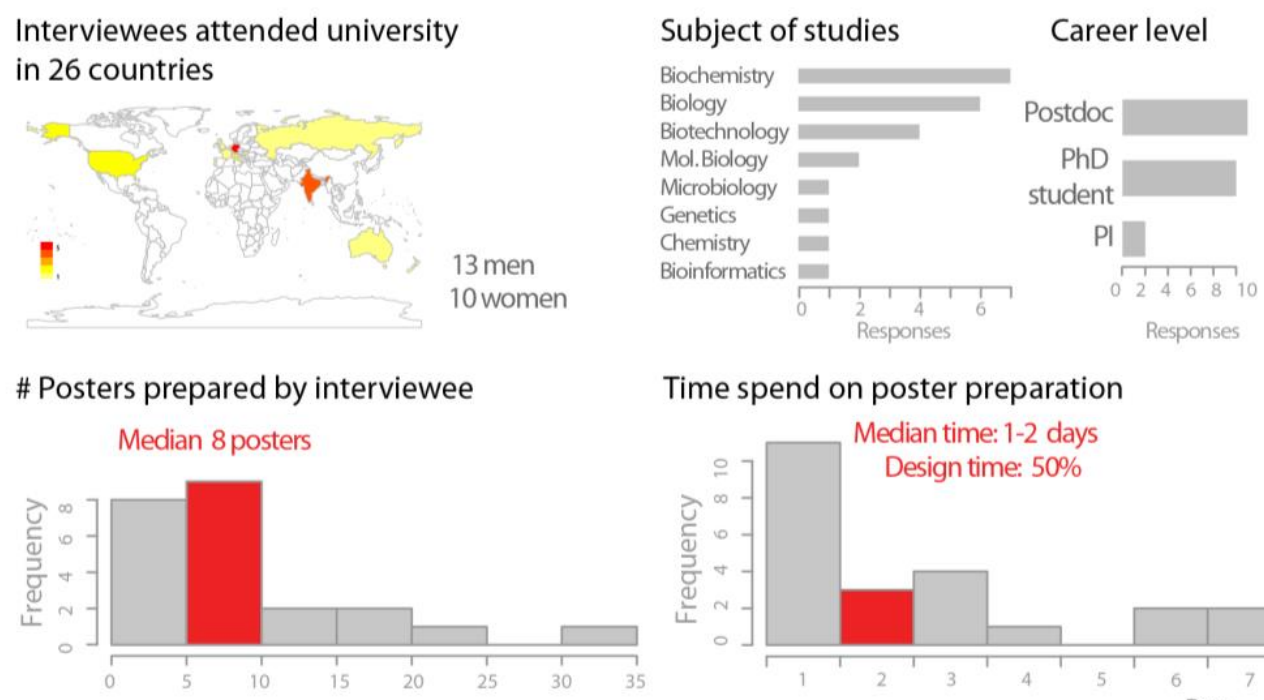

Time spend on poster preparation

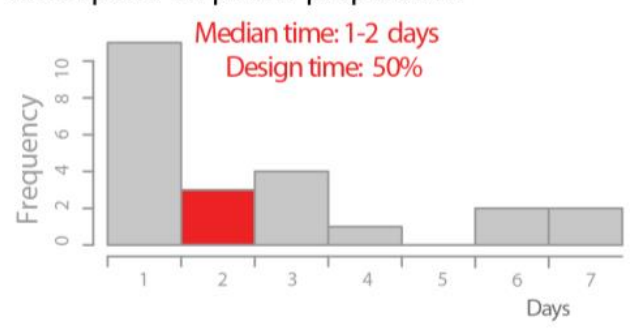

Poster making process

Software for poster preparation Of 23 interviewees
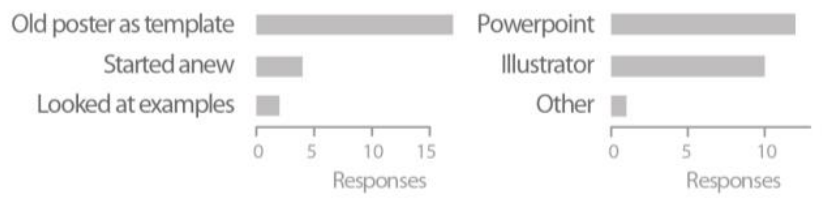


\section{Discussion}

The online survey and interviews show that scientists, across that career-stage, country of studies, and discipline, receive little training in information design or software for poster preparation. Further, feedback from colleagues/supervisors during poster drafting stage seems to be rare, but it is not clear if respondents failed to ask of if this is simply not practiced. These factors contribute to the poor legibility of posters, which in turn means that their communication potential is not fully exploited.

Three simple measures may effectively improve poster quality: 1) Many scientists reuse old posters, each laboratory should therefore have a legible and well-designed template. Some universities provide templates, or a custom template could be designed with professional help, and online templates are available (see Colin Purrington blog). 2) Laboratories should establish giving feedback on poster legibility (text size/abbreviations, consistent colours, clear reading direction, large/labelled images/charts etc, see references and blogs e.g. http://betterposters.blogspot.de/). Important, feedback should be offered on the draft, nobody wants to re-design a final poster. 3) Scientists could tremendously benefit from learning a vector-based graphic editor and principles of information design (see box). The widespread use of unsuitable software prolongs the poster preparation time and often results in pixelated content. Given that students in biomedicine prepare around 8 posters during their $\mathrm{PhD}$, this sums up to the sizable time commitment of 14-21 working days. As a sideeffect, software training would also improve preparation of manuscript figures and slides.

\section{Box 1: Useful resources and software}

Books about data visualization principles

- Edward Tufte (1983) The Visual Display of Quantitative Information

- Alberto Cairo (2016), The Truthful Art

- Alberto Cairo (2011), The Functional Art

- Dona M. Wong (2010) The Wall Street Journal Guide to Information Graphics

Software, vector graphics editors:

- Adobe Illustrator (MacOS, Windows)

- CorelDraw (MacOS, Windows)

- Inkscape (open source, MacOS, Windows)

- AffinityDesigner (MacOS, Windows) 


\section{References}

Boullata, JI, Mancuso, CE, 2007, A "How-To" Guide in Preparing Abstracts and Poster Presentations, doi.org/10.1177/0115426507022006641

Brown BS. Communicate your science!... Producing punchy posters. Trends Cell Biol. 1996;6:37-39. doi: 10.1016/0962-8924(96)81037-3.

Brown BS. Poster design—six points to ponder. Biochem. Educ. 1997;25:136-137. doi: 10.1016/S0307-4412(97)00045-9.

Erren TC, Bourne PE. Ten simple rules for a good poster presentation. PLoS Comput Biol. 2007; 3: 777- 778 .

Gundogan B, Koshy K, Kurar L, Whitehurst K. How to make an academic poster. Ann. Med. Surg. 2016;11:69-71. doi: 10.1016/j.amsu.2016.09.001.

Jambor, H 2017. https://helenajambor.wordpress.com/2017/11/23/how-to-win-aconference-prize/ and https://helenajambor.wordpress.com/2018/06/06/its-playtimebad-poster-bingo

Wolcott, TG http://www.sicb.org/newsletters/fa97nl/sicb/poster.html

\section{Acknowledgements}

I would like to thank Lorenzo Amabili and Iris Morgenstern for reading a draft manuscript. 